\title{
The Vocal Chorder
}

Probing stamina in an interactive artifact

through opera rehearsals.

\section{Describe what you made.}

The Vocal Chorder is a large interactive instrument that allows for full bodily interaction with music and visuals. By pushing, pulling, and leaning on steel wires, opera singers accompany their own voices and thereby experience embodiment and empowerment.

\section{Briefly describe the process} of how this was made.

The first incarnation of the Vocal Chorder had as its main goal to enable one of the authors - who is also an opera singer - to establish an autonomous, interactive embodied performance where visuals and accompaniment created a homogenous gestalt. The design was guided by the unique needs of the singer, explored through autobiographical design and on-stage material explorations, and later tested by other singers and audiences. The longterm development lasted more than 10 years and many incarnations of the software; however, the custom-built hardware remained the same.

What is the most important/ key/interesting thing about what you made?

As opera normally is rehearsed meticulously before the audience meets it, a rehearsal period is in fact an inquiry into the endurance of the materials, calling for what we have named

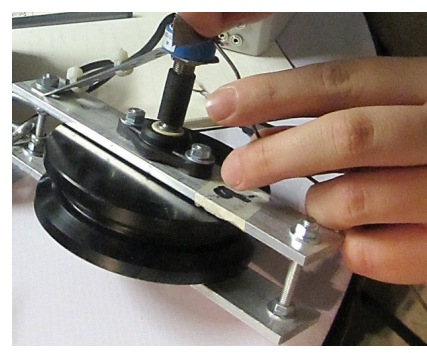

$\rightarrow$ One of eight sensors, consisting of wheel, housing, cabling, and potentiometer.

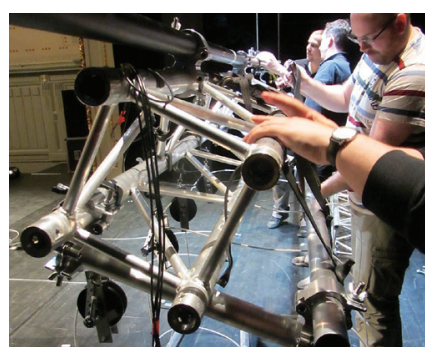

$\rightarrow$ Stamina and robustness probed through the many repetitions in the rehearsal phase leading up to a premiere.

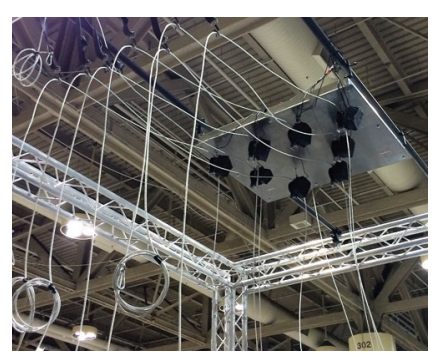

$\rightarrow$ Thick rubber bands fixed at the end of each wire roll over the wheels to turn the potentiometers, allowing for heavy-duty bodily interaction.

performative stamina. We explored the many ingoing components by designing for the extreme requirements for putting an interactive device on stage. We found that an almost ritual approach when handling materials such as computers, software, and sensors was essential.
What expertise Iskills and competences) did it require? Our team has deep knowledge of the procedures in opera productions, and it turned out the circumstances that are typical for operatic stage work-where reliability is probed through the many repetitions in the rehearsal phase leading up to a premiere-offered an excellent biotope for the thorough probing of artifacts.

\section{What materials and tools did} you use?

Our hardware development revolved around the novel use of eight potentiometers as sensors for bodily interaction. By turning the potentiometers, we could accurately affect changes in the music and visuals.

We needed to transform the movement of the body in space into a means for turning the potentiometers, which led us to try steel wires leading from the floor to the ceiling. They enabled the performer to stand and even dangle outside of the artifact while pushing and pulling the wires over the potentiometers. The potentiometers were therefore mounted in the ceiling on large wheels that allowed for the steel wires to roll them back and forth. Thick rubber bands were fixed at the end of each wire, letting them roll over the wheels to turn
Q Specs

Materials: Potentiometers housed in styrene wheels, rubber bands, steel wires connecting custom-built ceiling with floor lallowing for full-body interaction), voltage-to-midiconverter, loudspeakers, projectors

Tools (Software): Max/MSP, SuperCollider, Processing, Finale, Logic the potentiometers under the large pressure from the bodily interactions.

What is the one thing about making this that you would like to share with other makers?

In order to fine-tune a multifaceted digital instrument like this, we developed a procedure where artistic intuition and experience continuously influenced the technical development and vice versa. We named this process sensory digital intonation, whereby precise considerations on artistic expression within the sensory interaction happen. For example, carefully judging how far the wires are to be bent before a chord is sounding, or setting how multiple parameters that are affected by the same wire interplay with each other in an artistically viable way. Using sensory digital intonation we fine-tuned the artifact toward performing with it, balancing the many parameters in software and hardware against one another.

Carl Unander-Scharin University College of Opera and KTH Royal Institute of Technology (1) Åsa Unander-Scharin, Luleå University of Technology

(4) Kristina Höök, Mobile Life a KTH Royal Institute of Technology (4) Ludvig Elblaus, KTH Royal Institute of Technology 


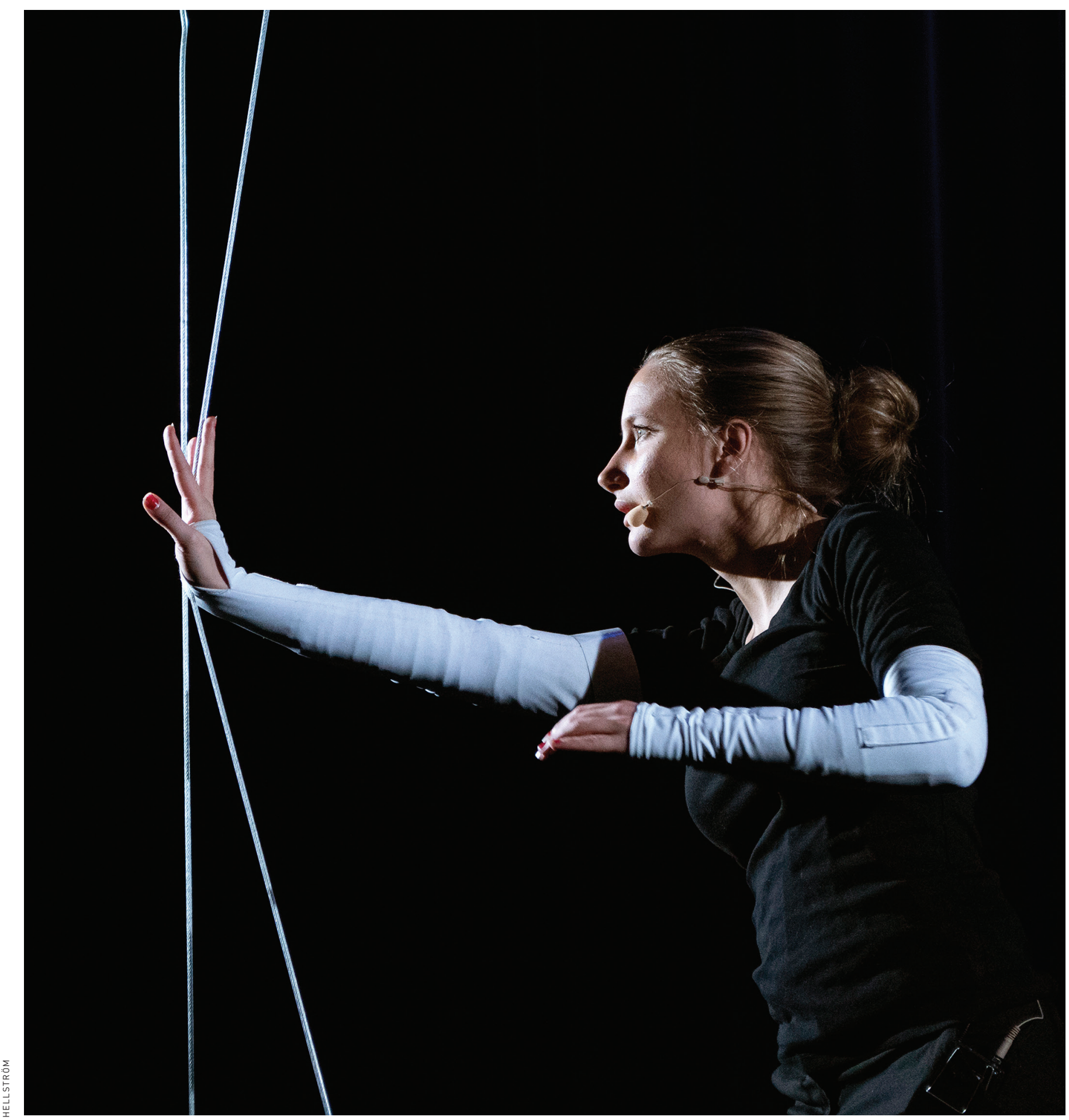

$\rightarrow$ Emilia Feldt performing with the Vocal Chorder. Liszt-Academy, Budapest, Hungary, 2014

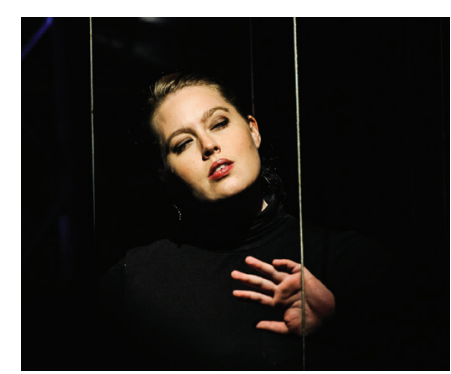

$\rightarrow$ Randi Røssakk performing with the Vocal Chorder in the KTH Reactor Hall, Stockholm, Sweden, 2011

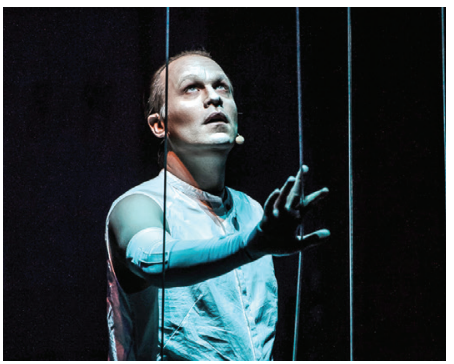

$\rightarrow$ Staffan Liljas performing with the Vocal Chorder, Cape Town, South Africa, 2013

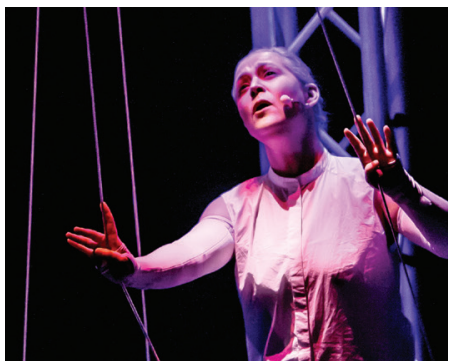

$\rightarrow$ Lisa Gustafsson interacting with the Vocal Chorder, Cape Town, South Africa, 2013

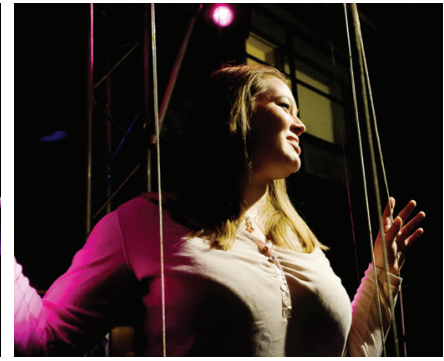

$\rightarrow$ Randi Røssakk interacting with the Vocal Chorder in the KTH Reactor Hall, Stockholm, Sweden, 2011 